\title{
Eyes wide shut: implied social presence, eye tracking and attention
}

\author{
Evan F. Risko - Alan Kingstone
}

Published online: 19 November 2010

(C) Psychonomic Society, Inc. 2010

\begin{abstract}
People often behave differently when they know they are being watched. Here, we report the first investigation of whether such social presence effects also influence looking behavior - a popular measure of attention allocation. We demonstrate that wearing an eye tracker, an implied social presence, leads individuals to avoid looking at particular stimuli. These results demonstrate that an implied social presence, here an eye tracker, can alter looking behavior. These data provide a new manipulation of social attention, as well as presenting a methodological challenge to researchers using eye tracking.
\end{abstract}

Keywords Attention - Eye tracking · Social cognition

\section{Introduction}

Cognitive psychology is becoming increasingly sensitized to the importance of social influences on attention. To date, studies of social attention have largely focused on questions regarding attention to social stimuli. For example, how do individuals use the gaze of others to direct their attention (e.g., Wilkowski, Robinson, \& Friesen, 2009), or how do individuals distribute their attention when social cues are present (e.g., Birmingham, Bischof, \& Kingstone, 2008; Zwickel \& Vo, 2010). In the present investigation, we

E. F. Risko $(\bowtie)$

Social and Behavioral Sciences, Arizona State University,

Glendale, AZ 85306, USA

e-mail: Evan.Risko@asu.edu

A. Kingstone

University of British Columbia,

Vancouver, Canada extend this line of investigation to how the broader social context can influence how attention is distributed.

Social presence

It is well known that the presence of others can alter one's own behavior (Aiello \& Svec, 1993; Bond \& Titus, 1983; Latane, 1981; Zajonc, 1965). For example, Satow (1975) demonstrated that individuals were more likely to donate money when another individual could see them. Interestingly, another individual does not actually need to be physically present to influence behavior (Aiello \& Svec, 1993; Putz, 1975; Van Rompay, Vonk, \& Frasen, 2009). Rather, it is enough for their presence to be implied. For example, Van Rompay et al. (2009) demonstrated that individuals who thought that they were being videotaped were more likely to help a confederate. As these examples suggest, the presence of others can lead individuals to alter their behavior in a manner that communicates a positive self-impression (Baumeister, 1982; Bond, 1982; Latane, 1981; Risko, Quilty, \& Oakman, 2006; Satow, 1975; Van Rompay et al., 2009). Here, we set out to determine whether a social presence can influence an individual's looking behavior in a similar manner.

Looking behavior

Studying where people look has become one of the standard vehicles for trying to understand attention. For example, researchers interested in attentional selection record where individuals look in scenes (e.g., Itti \& Koch, 2000; Underwood \& Foulsham, 2006). In addition, to better understand social attention, researchers have focused on biases in looking at social stimuli (e.g., Birmingham et al., 2008) and the characteristics of individual's looking 
behavior while engaged in social tasks (e.g., conversation; Keysar, Barr, Balin, \& Brauner, 2000). The principal means for studying looking behavior is eye tracking (Duchowski, 2005; Rayner, 1998). Eye trackers provide a moment-tomoment record of where an individual is looking and thus provide an indispensible tool for studying attention. Interestingly, eye trackers are essentially video cameras for the eyes, thus they could, in principle, act as an implied social presence. Indeed, eye trackers could be viewed as a rather invasive implied social presence. The eyes have long been considered "special" in terms of their ability to communicate our thoughts to others (e.g., Baron-Cohen, 1995). Thus, to have our eyes recorded could engage mechanisms similar to those engaged by a more traditional video camera or another person.

That said, one could reasonably argue that looking behavior is unlike any other kind of behavior demonstrated to be influenced by social presence (e.g., helping), in that looking behavior is less dependent on explicit or overt control. Our eyes, at least subjectively, seem to be where they need to be as we go about our interactions with the world. Indeed, researchers have demonstrated that individuals can be unaware that their eyes have moved (Belopolsky, Kramer, \& Theeuwes, 2008), and even when individuals are aware of the explicit need to look in a particular direction under certain circumstances, it can be difficult to do so (Munoz \& Everling, 2004; Theeuwes, Kramer, Hahn, \& Irwin, 1998). Against this background, it is unclear whether we should or should not expect that looking behavior be influenced by a social presence.

While the influence of social presence provides a novel environment for understanding social influences on attention, the idea that wearing an eye tracker could lead to changes in looking behavior also has important methodological implications. The potential influence of the act of observation on the behavior observed, while certainly not lost on psychologists (Adair, 1984), is not a pressing methodological issue in attention research. This fact could certainly be justified given the types of tasks typically employed; however, as researchers become more interested in the social dimension of attention, understanding the impact of the testing context will become increasingly important.

\section{Present investigation}

In order to determine if a social presence can influence looking behavior, we compared looking behavior when individuals knew their eyes were being watched (via an eye tracker) and when they did not (via a hidden camera). Participants took part in a "natural looking" task in which they performed a simple computer task alone in a testing room that contained objects typically found in an office.
After completing the computer task, participants remained alone in the room for a short amount of time. It was participants looking behavior during this free time that was the looking behavior of interest. This task was developed in an attempt to measure an individual's natural looking behavior (i.e., looking behavior exhibited when it is not explicitly the object of study; Tunnell, 1977). To covertly monitor looking behavior, a pinhole camera was hidden in the room.

To test whether wearing an eye tracker alters looking behavior in a manner consistent with social presence effects (i.e., impression management), we included a somewhat provocative stimulus (i.e., a swimsuit calendar) in the testing room. If social presence influences looking behavior, then individuals wearing an eye tracker should be less likely to look at the provocative stimulus than individuals not wearing an eye tracker.

\section{Experiment 1}

\section{Methods}

Participants Twenty-four undergraduates (17 females) from the University of British Columbia were paid $\$ 5$ each or received course credit to participate. Following the experiment, all participants were told about the hidden camera, at which point they had the option to consent or not to our use of that recorded information.

Apparatus and stimuli The Applied Science Laboratory MobileEye eye tracker was used and consists of a headmounted system with the glasses connected to a digital video recorder that records information about the eye and the scene. The pinhole camera was embedded in the swimsuit calendar (the provocative stimulus) located $90^{\circ}$ to the participant's right (relative to when they were facing the computer).

Procedure Participants were brought to the testing room and sat in front of a computer. In the no eye tracker condition participants were then instructed about how to perform the computer task (a 6-min cueing experiment). In the eye tracker condition, participants were fit with the eye tracker and a brief calibration (less than a minute) was conducted prior to being instructed about how to perform the computer task. The eye tracker manipulation was between subjects.

The participant performed the computer task alone and, once it ended, a screen was presented that informed participants that they should wait for the experimenter to return. The experimenter returned approximately $1 \mathrm{~min}$ after the computer task was over. 
Results

Two raters naïve to the purpose of the study coded looking behavior in 1-min clips of participants sitting alone in the room following the computer task. Raters coded whether participants looked at the camera (the camera was in the calendar). There was a high degree of agreement between raters, $\mathrm{K}=.91, p<.05$. When coders did not agree $(n=1)$ on whether or not the participant looked at the calendar, the data were removed leaving a final sample size of 23. A chi-square analysis with a single degree of freedom $(d f=1)$ was used here and in all following analyzes to compare the groups.

In the no eye tracker condition, $92 \%$ of the participants looked at the provocative stimulus while only $36 \%$ of the participants wearing an eye tracker did the same (see Fig. 1). This difference was statistically significant, $X^{2}=7.74, p<.05$, and is consistent with the idea that an implied social presence, in this case an eye tracker, can influence looking behavior.

\section{Experiment 2}

In Experiment 2, we repeated the conditions of Experiment 1 , but added a third condition in which participants wore the eye tracker but were instructed that it was turned off (non-operational eye tracker condition). We included this condition to control for any possible effect of the physical act of wearing an eye tracker. In addition, at the end of the study, participants were asked whether they "thought" or "felt" that their eyes were being tracked. These questions were included as a manipulation check (i.e., some participants might not believe that the eye tracker was off). If it is the knowledge that one's eyes are being tracked and not the physical act of wearing the eye tracker that is responsible for the results of Experiment 1, then individuals in the nonoperational eye tracker condition (who believe that the eye tracker is off) should be just as likely as individuals not wearing an eye tracker to look at the provocative stimulus.

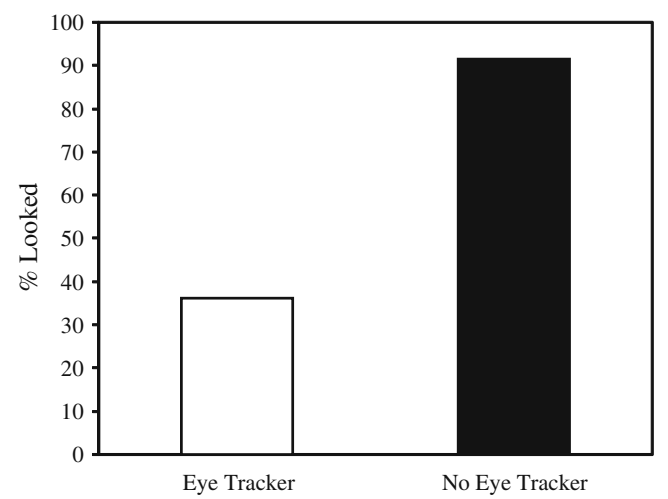

Fig. 1 Percentage of participants who looked at the provocative stimulus in Experiment 1
Methods

Participants Forty-five undergraduates (30 females) were paid $\$ 5$ each or received course credit to participate.

Apparatus, stimuli and procedure Identical to Experiment 1 save for the addition of the non-operational eye tracker condition. In this condition, participants were told that they had to wear the eye tracker but that it was not turned on. There was no calibration in this condition. After the free looking time, participants who were wearing the eye tracker were also given an exit survey asking them if they thought that or felt as if their eyes were being tracked.

\section{Results and discussion}

The data were handled as in Experiment 1. There was again a high degree of agreement between raters, $\mathrm{K}=.73, p<.05$, and coder disagreements were removed $(n=6)$ leaving a final sample size of 39. A number of participants in the non-operational eye tracker condition reported that they thought and/or felt as if their eyes were being tracked. As a result, we report results separately for individuals who thought/felt like their eyes were being tracked and those that did not.

In the no eye tracker condition, $100 \%$ of the participants looked at the provocative stimulus while only $21 \%$ of the participants wearing an eye tracker did the same (see Fig. 2). This difference was significant, $X^{2}=15.43, p<.05$, thus replicating Experiment 1's results.

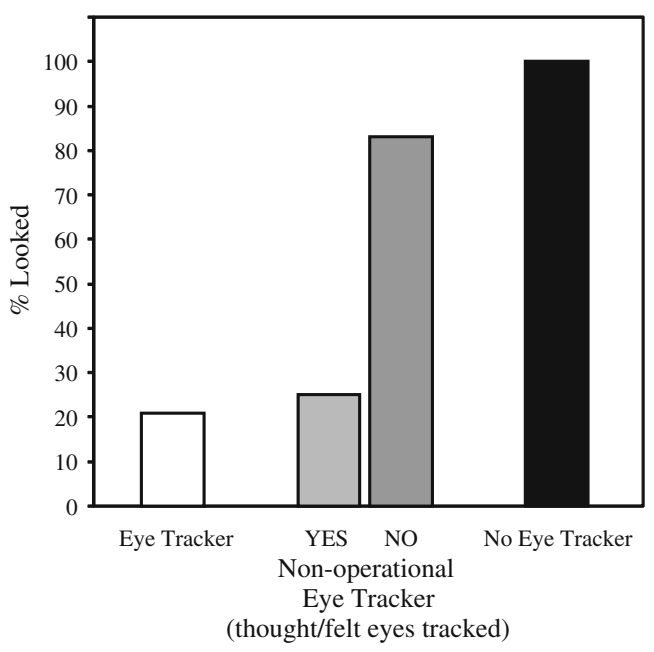

Fig. 2 Percentage of participants who looked at the provocative stimulus in Experiment 2. In the non-operational condition, the Yes label refers to participants who did think/feel that their eyes were being tracked and the No label refers to participants who did not think/ feel that their eyes were being tracked 
In the non-operational eye tracker condition, $8 / 14$ participants thought/felt that their eyes were being tracked (despite being told the eye tracker was off) and only $25 \%$ of them looked at the swimsuit calendar. The remaining participants believed that their eyes were not being tracked (i.e., the manipulation was effective) and $83 \%$ of these participants looked at the calendar. Critically, the looking behavior of this group was the same as those not wearing an eye tracker, $X^{2}=1.95, p>.05$, and significantly different from those wearing an eye tracker that they were told was on, $X^{2}=6.71, p<.05$. Thus, it is the mere belief that one's eyes are being tracked, or not, that leads to alterations in one's looking behavior.

\section{Experiment 3}

Thus far, our working assumption has been that individuals do not look at the provocative stimulus when they believe their eyes are being monitored because the eye tracker serves as an implied social presence that leads participants to modify their natural looking behavior to create a positive self-impression. Looking at a neutral calendar should not negatively impact an individual's self-impression, and therefore in Experiments $3 \mathrm{a}$ and $3 \mathrm{~b}$, we replaced the provocative calendar with a neutral calendar (see Fig. 3).

\section{Methods}

Participants In Experiment 3a, 24 (19 females), and in Experiment $3 \mathrm{~b}, 23^{1}$ undergraduates (14 females), were paid $\$ 5$ each or received course credit to participate.

Apparatus, stimuli and procedure These were identical to Experiment 1 with the exception that the swimsuit calendar was replaced by a neutral calendar. The neutral calendar consisted of natural landscapes in Experiment $3 \mathrm{a}$ and snowboarders in Experiment $3 \mathrm{~b}$.

\section{Results and discussion}

The data were handled as in the previous experiments. There was a high degree of agreement between raters in both Experiment $3 \mathrm{a}, \mathrm{K}=.75, p<.05$, and Experiment $3 \mathrm{~b}$, $\mathrm{K}=.77, p<.05$, and coder disagreements were removed from Experiment $3 \mathrm{a}(n=3)$ and $3 \mathrm{~b}(n=2)$ leaving a final sample size of 21 in both experiments.

\footnotetext{
${ }^{1}$ One participant was lost post-data collection because of an error in coding.
}

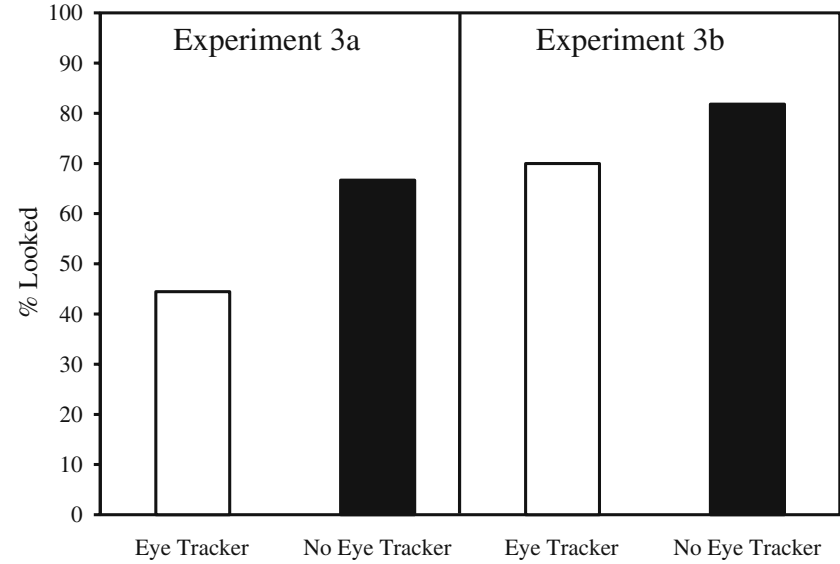

Fig. 3 Percentage of participants who looked at the neutral stimulus in Experiment $3 \mathrm{a}($ left $)$ and $3 \mathrm{~b}($ right $)$

\section{Experiment 3a}

In the no eye tracker condition, $66 \%$ of the participants looked at the calendar and $45 \%$ of the participants wearing an eye tracker did the same. This difference was not significant, $X^{2}=1.04, p>.05$, indicating that wearing an eye tracker did not influence the likelihood that participants would look at a neutral stimulus.

\section{Experiment 3b}

In the no eye tracker condition, $82 \%$ of the participants looked at the calendar and $70 \%$ of the participants wearing an eye tracker did the same. This difference was not significant, $X^{2}=0.40, p>.05$, indicating again that wearing an eye tracker did not influence the likelihood that participants would look at a neutral stimulus. These results can be contrasted with the large differences found when the same stimulus, in the same location, in the same task was provocative.

\section{Combined analysis}

In Experiments $3 \mathrm{a}$ and $3 \mathrm{~b}$, there was a small non-significant trend suggesting that individuals wearing an eye tracker might be less likely to look at a neutral calendar. To provide a more powerful analysis, the data from Experiments $3 \mathrm{a}$ and $3 \mathrm{~b}$ were combined. The small difference was still not significant, $X^{2}=1.20, p>.05$. To provide additional evidence for the interpretation of Experiments 1 and 2 in terms of a social presence effect, we conducted two analyses comparing looks within the eye tracker and no eye tracker conditions across the different calendar types (i.e., provocative vs neutral calendar). When participants were wearing an eye tracker, they were significantly less likely to look at the provocative calendar than the neutral 
calendar, $X^{2}=3.99, p<.05$. In addition, when individuals were not wearing an eye tracker, participants were significantly more likely to look at the provocative calendar than the neutral calendar, $X^{2}=4.21, p<.05$. This crossover demonstrates that the effect of wearing an eye tracker on looking behavior is more pronounced when the stimulus was socially charged. ${ }^{2}$

\section{General discussion}

The reported experiments demonstrate clearly that an implied social presence, in this case an eye tracker, can influence looking behavior. Individuals wearing an eye tracker alter their natural looking behavior in a manner consistent with a form of impression management. Specifically, in Experiments 1 and 2, participants wearing an eye tracker were much less likely to look at a provocative stimulus than individuals not wearing an eye tracker. In Experiments $3 \mathrm{a}$ and $3 \mathrm{~b}$, when the looked at stimulus was neutral, there was no difference between these two groups. Experiments 2, $3 \mathrm{a}$ and $3 \mathrm{~b}$ also demonstrate that the reduction in looking toward a provocative stimulus when individuals are wearing an eye tracker is not due to the physical act of wearing the eye tracker. If it were, then we would have expected to find equivalent effects of wearing an eye tracker on looking behavior when participants were told the eye tracker was off (and believed it was) and when the provocative stimulus was replaced by a neutral stimulus. Rather, it appears that it is the knowledge that one's eyes are being watched that alters looking behavior.

\section{Implications for social attention}

The demonstration that an implied social presence can influence looking behavior further extends work on the effects of social presence in general (Baumeister, 1982; Latane, 1981; Satow, 1975; Zajonc, 1965) and implied social presence in particular (Aiello \& Svec, 1993; Putz, 1975; Van Rompay et al., 2009). The extension to looking behavior also provides a clear demonstration of the influence of the social context on attention, thus broadening current work on social attention beyond responses to social stimuli. In the present case, a particular stimulus is attended to differently based on the larger social context (i.e., being alone vs being watched). Interestingly, this kind of contextual influence represents the bedrock of many social phenomena that are now coming into the focus of social

\footnotetext{
${ }^{2}$ We also conducted a combined analysis separately for females and males. The patterns were the same across gender. That said, there were relatively few males, which makes any strong claim about the lack of a gender effect tentative.
}

attention researchers (Crosby, Monin, \& Richardson, 2008). For example, how does social standing, social roles, or social identities influence the distribution of attention (Levine, Resnick, \& Higgins, 1993)? Pursuing these questions, amongst others, will aid in moving social attention research into the rich social environment in which attention operates on a day-to-day basis (Hutchins, 1995; Kingstone, 2009).

\section{Methodological implications}

The finding that wearing an eye tracker can alter looking behavior serves as an important reminder that the act of experimental observation can in some cases influence the behavior observed. At the most basic level, the present experiments demonstrate that (1) individuals are sensitive to their eyes being monitored (i.e., there is "eye tracker awareness"), and (2) participants are willing and able to modulate their looking behavior in response to this knowledge. These results represent an important methodological challenge for researchers interested in investigating attention in social contexts where social pressures could play a major role. The potential that eye tracking could influence looking behavior beyond putatively social tasks or manipulations should also not be ignored (i.e., the testing context is itself a social context; Hertwig \& Ortman, 2001).

\section{Natural behavior approach}

The present results also speak to the utility of studying natural behavior (Cialdini, 2009; Hutchins, 1995; Kingstone, Smilek, \& Eastwood, 2008; Tunnell, 1977). It was only through contrasting (more) natural looking behavior with looking behavior in the context of an eye tracking experiment that the influence of the method of study (i.e., eye tracking) became apparent. As cognitive researchers become more concerned with issues of embodiment (Pfiefer \& Bongard, 2006), situatedness (Robbins \& Aydede, 2009) and the distributed nature of cognition (Hutchins, 1995), a natural behavior approach will become increasingly important as a complement to more traditional approaches.

\section{Conclusion}

The present results demonstrate that an implied social presence can have a profound influence on looking behavior. This result provides a novel vantage point from which to view social influences on attention while also presenting an important methodological challenge to researchers using eye tracking in social settings. 
Acknowledgments This work was supported by a Natural Sciences and Engineering Research Council of Canada (NSERC) Postdoctoral Fellowship and a Killam Postdoctoral Fellowship to E.F.R. and NSERC operating grants to A.K.

\section{References}

Adair, J. G. (1984). The Hawthorne effect: A reconsideration of the methodological artifact. The Journal of Applied Psychology, 69, 334-345. doi:10.1037/0021-9010.69.2.334

Aiello, J. R., \& Svec, C. M. (1993). Computer monitoring and work performance: Extending the social facilitation framework to electronic presence. Journal of Applied Social Psychology, 23, 537-548. doi:10.1111/j.1559-1816.1993.tb01102.x

Baron-Cohen, S. (1995). Mindblindness: An essay on autism and theory of mind. Cambridge, MA: MIT Press.

Baumeister, R. F. (1982). A self-presentational view of social phenomena. Psychological Bulletin, 91, 3-26. doi:10.1037/0033-2909.91.1.3

Belopolsky, A. V., Kramer, A. F., \& Theeuwes, J. (2008). The role of awareness in processing of oculomotor capture: Evidence from event-related potentials. Journal of Cognitive Neuroscience, 20, 1-13. doi:10.1162/jocn.2008.20161

Birmingham, E., Bischof, W. F., \& Kingstone, A. (2008). Gaze selection in complex social scenes. Visual Cognition, 15, 341355. doi:10.1080/13506280701434532

Bond, C. F. (1982). Social facilitation: A self-presentational view. Journal of Personality and Social Psychology, 42, 1042-1050.

Bond, C. E., \& Titus, L. J. (1983). Social facilitation: A meta-analysis of 241 studies. Psychological Bulletin, 94, 265-292. doi:10.1037/0022-3514.42.6.1042

Cialdini, R. B. (2009). We have to break up. Perspectives on Psychological Science, 4, 5-6. doi:10.1111/j.1745-6924.2009.01091.x

Crosby, J. R., Monin, B., \& Richardson, D. C. (2008). Where do we look during potentially offensive behavior? Psychological Science, 19, 226-228. doi:10.1111/j.1467-9280.2008.02072.x

Duchowski, A. T. (2005). A breadth-first survey of eye-tracking applications. Behavior Research Methods, Instruments and Computing, 34, 455-470.

Hertwig, R., \& Ortmann, A. (2001). Experimental practices in economics: A methodological challenge for psychologists? The Behavioral and Brain Sciences, 24, 383-451.

Hutchins, E. (1995). Cognition in the Wild. Cambridge, MA: MIT Press.

Itti, L., \& Koch, C. (2000). A saliency-based search mechanism for overt and covert shifts of visual attention. Vision Research, 40, $1489-1506$.

Keysar, B., Barr, D. J., Balin, J. A., \& Brauner, J. S. (2000). Taking perspective in conversation: The role of mutual knowledge in comprehension. Psychological Science, 11, 32-38. doi:10.1111/ 1467-9280.00211

Kingstone, A. (2009). Taking a real look at social attention. Current Opinion in Neurobiology, 19, 52-56. doi:10.1016/j.conb.2009.05.004
Kingstone, A., Smilek, D., \& Eastwood, J. D. (2008). Cognitive ethology: A new approach for studying human cognition. British Journal of Psychology, 99, 317-340. doi:10.1348/ $000712607 \mathrm{X} 251243$

Latane, B. (1981). The psychology of social impact. The American Psychologist, 36, 343-356. doi:10.1037/0003-066X.36.4.343

Levine, J. M., Resnick, L. B., \& Higgins, E. T. (1993). Social foundations of cognition. Annual Review of Psychology, 44, 585612. doi:10.1146/annurev.ps.44.020193.003101

Munoz, D. P., \& Everling, S. (2004). Look away: The anti-saccade task and the voluntary control of eye movements. Nature Reviews. Neuroscience, 5, 218-228. doi:10.1038/nrn1345

Pfeifer, R., \& Bongard, J. C. (2006). How the body shapes the way we think - A new view of intelligence. Cambridge, MA: MIT Press.

Putz, V. R. (1975). The effects of different modes of supervision on vigilance behaviour. British Journal of Psychology, 66, $157-160$.

Rayner, K. (1998). Eye movements in reading and information processing: 20 years of research. Psychological Bulletin, 124, 372-422. doi:10.1037/0033-2909.124.3.372

Risko, E. F., Quilty, L. C., \& Oakman, J. M. (2006). Socially desirable responding on the web: Investigating the candor hypothesis. Journal of Personality Assessment, 87, 269-276. doi:10.1207/ s15327752jpa8703_08

Robbins, P., \& Aydede, M. (Eds.). (2009). Cambridge handbook of situated cognition. New York, NY: Cambridge University Press.

Satow, K. (1975). Social approval and helping. Journal of Experimental Social Psychology, 11, 501-509. doi:10.1016/0022-1031 (75)90001-3

Theeuwes, J., Kramer, A. F., Hahn, S., \& Irwin, D. E. (1998). Our eyes do not always go where we want them to go: Capture of the eyes by new objects. Psychological Science, 9, 379-385. doi:10.1111/1467-9280.00071

Tunnell, G. (1977). Three dimensions of naturalness: An expanded definition of field research. Psychological Bulletin, 84, 426-477. doi: $10.1037 / 0033-2909.84 .3 .426$

Underwood, G., \& Foulsham, T. (2006). Visual saliency and semantic incongruency influence eye movements when inspecting pictures. The Quarterly Journal of Experimental Psychology, 59, 1931-1949. doi:10.1080/17470210500416342

Van Rompay, T. J. L., Vonk, D. J., \& Fransen, M. L. (2009). The eye of the camera: Effects of security cameras on prosocial behavior. Environment and Behavior, 41, 60-74. doi:10.1177/ 0013916507309996

Wilkowski, B. M., Robinson, M. D., \& Friesen, C. (2009). Gazetriggered orienting as a tool of the belongingness self regulation system. Psychological Science, 20, 495-501. doi:10.1111/j.14679280.2009.02321.x

Zajonc, R. B. (1965). Social facilitation. Science, 149, 269-274. doi:10.1126/science.149.3681.269

Zwickel, J., \& Vo, M. L. H. (2010). How the presence of persons biases eye movements. Psychonomic Bulletin \& Review, 17, 257 262. doi:10.3758/PBR.17.2.257 